\title{
Planetary candidates around the pulsating sdB star KIC 5807616 considered doubtful
}

\author{
J. Krzesinski \\ Mt. Suhora Observatory, Pedagogical University of Cracow, ul. Podchorążych 2, 30-084 Cracow, Poland \\ e-mail: jk@astro.as.up.krakow.pl
}

Received 17 April 2015 / Accepted 12 June 2015

\begin{abstract}
Context. It has been suggested that two weak signals observed in the low frequency region of the Fourier transform amplitude spectra from the KIC 5807616 Q5-Q8 data can be interpreted as a result of the light reflection from planets orbiting the host star. Ever since the last results on KIC 5807616 were presented, the Kepler spacecraft has collected over two years of additional data, which we analysed using asteroseismological methods.

Aims. To verify and improve on previous results, we used the Q 5-Q 17 Kepler data to identify pulsational modes, determine multiplet splitting, and to re-analyse the low frequency region between $33-49 \mu \mathrm{Hz}$ where two frequencies, claimed as the planetary signature, were found.

Methods. Since Fourier transform amplitude spectra of the KIC 5807616 data do not show any clear multiplets, we used two stable acoustic modes to determine the theoretical width of gravity mode multiplets and their splittings. The period spacing and histograms of common multiplet component separations were used to identify pulsation modes and the observed gravity mode splittings. In the low frequency region, we analysed the amplitude variations of two planetary signature frequencies over the whole observing run.

Results. We determined the rotational period of the star from the splittings. Analysis of the low frequency region shows that the amplitude and frequency change of the signals found there have similar characteristics to other gravity modes.

Conclusions. New data allow for identifying gravity modes in a limited period range, as well as better rotational period estimations. We suggest that the so-called planetary signature frequencies found in previous work might instead be pulsation modes visible beyond the cut-off frequency of the star.
\end{abstract}

Key words. subdwarfs - stars: rotation - asteroseismology - planetary systems

\section{Introduction}

KIC 5807616 is a pulsating B-type hot subdwarf (sdB), also known as KPD 1943+4058, from the extreme horizontal branch of the H-R diagram. Its preliminary Q 2.3 data from the Kepler exploratory phase was initially analysed by Østensen et al. (2010) and Reed et al. (2010), while Van Grootel et al. (2010) derived KIC 5807616's stellar parameters from spectroscopic observations. The star pulsates in both gravity $(g-)$ and pressure $(p-)$ modes, therefore it is a hybrid sdBV (Schuh et al. 2005; Baran et al. 2005). Its $27730 \pm 270 \mathrm{~K}$ effective temperature, $\log g=5.52 \pm 0.03$ surface gravity, and $0.496 \pm 0.002 M_{\odot}$ mass (Van Grootel et al. 2010) are typical of this class of objects.

The following Q 5-Q 8 quarters of KIC 5807616 observing data have been analysed by Charpinet et al. (2011). They find that the star has a rich pulsation spectrum, but only two $p$-modes and several $g$-modes (one higher than $l=2$ ) were identified. The reason for the limited success of mode identification was the lack of clear multiplet structures in the Lomb-Scargle periodograms of the KIC 5807616 light curve. Despite having a limited number of multiplets, Charpinet et al. (2011) were able to derive the rotational period of the star as 39.23 days, from the splitting of two $p$-mode multiplet components. Finally, Charpinet et al. (2011) argue that the weak modulations in the low frequency region can be explained by the existence of planets around KIC 5807616. Using the cut-off frequency (Hansen et al. 1985) to determine the limit for pulsational periods, as well as careful analysis of the light curve, Charpinet et al. (2011) conclude that 5.7625 and $8.2293 \mathrm{~h}$ periods visible in their periodogram come from the reflection effect of two planets orbiting KIC 5807616. The analysis of the amplitude ratio of multiplet components allowed them to determine the inclination of the star's rotational axis to be $\sim 65^{\circ} \pm 10^{\circ}$ and in this way established the orbital plane inclination of the planetary system.

Since then the Kepler spacecraft has collected eight quarters of KIC 5807616 observing data, and in this work we present the results of the Fourier transform (FT) amplitude spectra analysis of the whole Q 5-Q 17 light curve, as well as of four shorter data sets (seasons). All significant FT signals were also analysed by means of the high resolution (200 days) time-frequency or running FT. We focused on the mode identification, multiplet structures, and the low frequency region, where the signatures of two planetary candidates were claimed. A nearly three times longer set of data allowed us to change some preliminary estimates and to shed new light on the interpretation of planetary signature frequencies.

\section{Data and mode identification}

During the exploratory phase, KIC 5807616 was observed in the second quarter for one month $(\mathrm{Q} 2.3)$ in the short cadence data mode of $58.89 \mathrm{~s}$ exposure time and for three months in the long cadence data mode of $30 \mathrm{~min}$ exposure time. Following a six month gap, the star has been observed continuously from Q 5 up to Q 17, i.e. until the failure of the second Kepler reaction wheel had occurred. We used this continuous set of short cadence data (1114.5 days or $\sim 3$ years) for the FT analysis. 
To avoid possible flux contamination by the neighbouring stars in the field, we downloaded the short cadence data target pixel Kepler data from the Barbara A. Mikulski Archive for Space Telescopes (MAST). The fluxes were then extracted only from non-contaminated pixels and preprocessed using cotrending basis vectors (see Kepler Data Release 12 Notes). Points outlying more than $3 \sigma$ from the running mean of ten points were removed from the data, along with the remaining trends (by fitting spline functions). Since Kepler pixel data before Q 15 suffer a time problem (Kepler Data Release 21 Notes), all data points were accordingly time-corrected. The fluxes were then converted into ppt units (parts per thousand) and used to calculate the FT of the whole light curve and FTs of the seasonal data resulting from the division of the whole 1114 day light curve into four approximately equal ( 280 days) parts (seasons 1, 2, 3, and 4). Following standard procedures, the pulsation frequencies were selected from the FTs and simultaneously fitted to the time series data using nonlinear least-squares techniques and prewhitened from the data. The prewhitening process was repeated until most frequencies with a signal above the $4 \sigma$ detection threshold of $0.01743 \mathrm{ppt}$ (calculated for the whole data within $0-600 \mu \mathrm{Hz}$ ) were removed.

For the low frequency region $(10-100 \mu \mathrm{Hz})$ and a couple of frequencies, we also used a local detection threshold of $0.015 \mathrm{ppt}$. The detection thresholds for seasonal data were $0.037 \mathrm{ppt}$ (calculated between $0-600 \mu \mathrm{Hz}$ where all of the high amplitude modes are present) and $0.023 \mathrm{ppt}$ (above $600 \mu \mathrm{Hz}$ ). The frequency resolution calculated as $1.5 / \mathrm{T}$ ( $\mathrm{T}-$ the time span of the data) for Q 5-Q 17 was $\sim 0.01 \mu \mathrm{Hz}$, allowing for very close frequencies to be fitted. The FT resolutions of any of the seasonal data were better than the 200 days running FTs $(0.06$ and $0.09 \mu \mathrm{Hz}$, respectively). Owing to the amplitude, phase, or frequency variations, several of the frequencies could not be prewhitened. Therefore, some of them were manually added later to the fitted frequency list. In total, we were able to fit over 190 frequencies to the light curve of KIC 5807616.

Because the FT of the KIC 5807616 light curve does not show clear multiplet structures, less than half of the fitted frequencies were useful for mode identification. The only two clearly visible multiplets, with well separated and stable components, were found in the $p$-mode frequency region at 3431.8383 and $3447.2453 \mu \mathrm{Hz}$. Therefore, for mode identification we used widths and general appearance of the multiplets in the FTs of the whole and seasonal data, as well as in high resolution running FTs calculated within a narrow $\pm 1.7 \mu \mathrm{Hz}$ frequency range around multiplets. In most cases, their internal structure was ignored.

\subsection{Period spacing fit}

Since there are no clear multiplet structures in the $g$-mode frequency region, we decided to visually choose central frequency positions of multiplets from the whole data FT, instead of relying on the fit frequencies. Then, we converted them to periods, for the period spacing fit. Because higher modes are expected to be reduced in amplitude by geometric cancellations (Dziembowski 1977), the selection of periods for spacing fits started with the assumption that the largest amplitude periods are $l=1$ mode, and the rest were fitted as $l=2$ modes. When all high amplitude modes were assigned, we used a deviation criteria. Modes that deviated noticeably from one-mode period spacing were fitted with another set of periods and assigned to the set in which their deviations from the period spacing were smaller. This preliminary assignment of modes was changed afterwards based on a

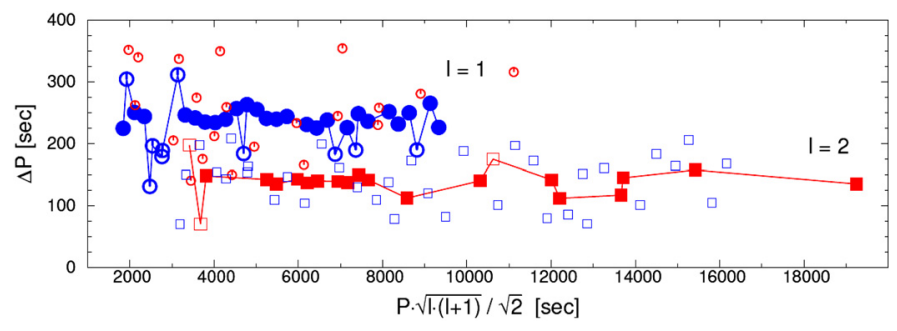

Fig. 1. $\Delta P$ vs. reduced period (divided by $\sqrt{2}$ ) diagram for $l=1$ (large blue filled and open circles) and $l=2$ (large red filled and open squares). Large open symbols represent periods that deviated more than $30 \mathrm{~s}$ from the period spacing fit. Small symbols represent $l=1$ (blue small open squares) or $l=2$ (red small open circles) calculated with the period spacing fit of another mode.

visual inspection of $l=1$ and $l=2$ multiplet structure splittings and widths. For that purpose, following Charpinet et al. (2011), we determined the theoretical $g$-mode frequency splittings from two clear $p$-mode multiplets mentioned earlier (see also chapter Multipletsandrotation below). This way we estimated $g$-mode splittings equal to 0.129 and $0.215 \mu \mathrm{Hz}$ for $l=1, l=2$ modes, respectively, and used them for mode identification. In Fig. 1 we present final period spacing $(\Delta P)$ vs. reduced period $(\mathrm{P}) \mathrm{di}$ agram for period spacing fits $\Delta P_{l=1}=241.48 \pm 0.26 \mathrm{~s}$ and $\Delta P_{l=2}=139.43 \pm 0.15 \mathrm{~s}$. Since the reduced period for $l=1$ is $P \cdot \sqrt{l \cdot(l+1)}=P \cdot \sqrt{2}$ and for $l=2$ modes, $P \cdot \sqrt{2 \cdot 3}$, only $l=2$ modes periods were multiplied by $\sqrt{3}$ to avoid large numbers in Fig. 1. Some of the modes can be classified equally well as $l=1$ or $l=2$ modes. There are also a few modes that could be classified as $l=3$, but we lack stronger evidence of that.

Using period spacing one can identify modes between $\sim 2000$ and $\sim 12000 \mathrm{~s}$. For longer periods, the spacing becomes comparable to the width of multiplets, and period spacing fit does not work accurately. For shorter periods the scatter of spacings between consecutive periods varies too much to rely only on the period spacing fit. Therefore, without clear multiplets we could identify only 56 modes ( $35 l=1$ and $21 l=2$ modes) in a narrow range of periods. Table 1 summarizes our identification efforts. We note that the first period $11111.8 \mathrm{~s}(89.99421 \mu \mathrm{Hz})$ in Table 1 identified as $l=2$ modes falls below the $l=2105.69 \mu \mathrm{Hz}$ cutoff frequency of gravity modes determined by Charpinet et al. (2011) (see the discussion of the cut-off frequency in Sect. 4 below). Since most of the fit frequencies can only be used for some statistical purposes (for example an average $l=1$ or $l=2$ multiplet component splitting determination), we decided to show only those frequencies that correspond to visually determined positions of the multiplet centres. The amplitudes given at these frequencies correspond to the amplitude of the highest peak of a given multiplet visible in the FT of the whole time series data.

In theory, the equal period spacing of gravity modes can be disturbed by mode trapping because of the transition zone between the hydrogen envelope and the helium mantle of the sdBV star (and perhaps by the transition zone between the mantle and the core, Charpinet et al. 2013). The deviations of trapped modes from equal period spacing make $\Delta P$ vs. $P$ patterns dependent on the hydrogen mass and in some cases can be used to estimate hydrogen masses of sdBV stars (Krzesinski et al. 2014). However, the analysis of KIC $5807616 \Delta P$ vs. $P$ diagrams in this regard has proved inconclusive.

The visibility of $\mathrm{m}$ componentes depends on the inclination of the star's rotation axis (Randall et al. 2005). Assuming that all component intrinsic amplitudes have the same level of 
J. Krzesinski: Planetary candidates around the pulsating sdB star KIC 5807616 considered doubtful

Table 1. Central frequencies of 56 multiplets used for mode identification in the $g$-mode frequency region between $89.99-542,17 \mu \mathrm{Hz}$.

\begin{tabular}{|c|c|c|c|c|c|c|c|c|c|}
\hline No & $\begin{array}{c}\text { Frequency } \\
{[\mu \mathrm{Hz}]}\end{array}$ & $\begin{array}{r}\text { Period } \\
{[\mathrm{s}]} \\
\end{array}$ & $\begin{array}{c}\text { Amplitude } \\
\text { [ppt] }\end{array}$ & $\begin{array}{c}\text { Mode } \\
\text { ID }\end{array}$ & No & $\begin{array}{c}\text { Frequency } \\
{[\mu \mathrm{Hz}]}\end{array}$ & $\begin{array}{r}\text { Period } \\
{[\mathrm{s}]} \\
\end{array}$ & $\begin{array}{c}\text { Amplitude } \\
\text { [ppt] }\end{array}$ & $\begin{array}{c}\text { Mode } \\
\text { ID }\end{array}$ \\
\hline 1 & 89.99421 & 11111.826 & 0.02294 & 2 & 29 & 212.71181 & 4701.197 & 0.06234 & 1,2 \\
\hline 2 & 107.11435 & 9335.817 & 0.04154 & 1 & 30 & 220.61806 & 4532.721 & 0.12966 & 1 \\
\hline 3 & 109.53692 & 9129.342 & 0.03185 & 1,2 & 31 & 225.95486 & 4425.663 & 0.01648 & 2 \\
\hline 4 & 112.88437 & 8858.622 & 0.02221 & 2,1 & 32 & 232.64699 & 4298.358 & 0.01859 & 2 \\
\hline 5 & 113.47789 & 8812.289 & 0.06403 & 1,2 & 33 & 234.25694 & 4268.817 & 0.12260 & 1 \\
\hline 6 & 115.86227 & 8630.938 & 0.01974 & 1 & 34 & 241.38194 & 4142.812 & 0.02457 & 2 \\
\hline 7 & 119.45602 & 8371.282 & 0.04908 & 1 & 35 & 248.31713 & 4027.108 & 0.33761 & 1 \\
\hline 8 & 122.64815 & 8153.405 & 0.03241 & 1 & 36 & 249.68403 & 4005.062 & 0.17989 & 2 \\
\hline 9 & 126.09375 & 7930.607 & 0.02665 & 2 & 37 & 264.10185 & 3786.418 & 0.56119 & 1 \\
\hline 10 & 126.68403 & 7893.655 & 0.02500 & 2,1 & 38 & 268.61690 & 3722.774 & 0.04036 & 2 \\
\hline 11 & 130.70370 & 7650.893 & 0.04301 & 1 & 39 & 278.98264 & 3584.452 & 0.01741 & 2 \\
\hline 12 & 134.73264 & 7422.107 & 0.02116 & 1,2 & 40 & 281.56250 & 3551.609 & 0.02607 & 1 \\
\hline 13 & 135.80903 & 7363.281 & 0.13129 & 1,2 & 41 & 290.18056 & 3446.130 & 0.07724 & 2 \\
\hline 14 & 139.70139 & 7158.125 & 0.02675 & 1 & 42 & 301.66435 & 3314.943 & 0.89456 & 1 \\
\hline 15 & 141.94097 & 7045.182 & 0.02681 & 2 & 43 & 316.01157 & 3164.441 & 0.02172 & 2 \\
\hline 16 & 144.18634 & 6935.470 & 0.03936 & 2 & 44 & 318.60532 & 3138.680 & 0.05389 & 1 \\
\hline 17 & 145.48611 & 6873.508 & 0.02996 & 1,3 & 45 & 329.79398 & 3032.196 & 0.05255 & 2 \\
\hline 18 & 149.53472 & 6687.410 & 0.03506 & 1 & 46 & 360.42824 & 2774.477 & 0.23468 & 1 \\
\hline 19 & 155.44676 & 6433.071 & 0.03272 & 1 & 47 & 361.72338 & 2764.543 & 0.17945 & 1 \\
\hline 20 & 161.36111 & 6197.280 & 0.04185 & 1 & 48 & 393.61343 & 2540.564 & 0.61576 & 1 \\
\hline 21 & 163.18750 & 6127.920 & 0.05143 & 2,3 & 49 & 403.98495 & 2475.340 & 0.11790 & 1,2 \\
\hline 22 & 167.83796 & 5958.128 & 0.79436 & 2,1 & 50 & 426.13542 & 2346.672 & 0.32189 & 1 \\
\hline 23 & 174.60185 & 5727.316 & 0.38963 & 1 & 51 & 454.31481 & 2201.117 & 0.01370 & 2 \\
\hline 24 & 182.43519 & 5481.399 & 0.02705 & 1 & 52 & 470.92361 & 2123.487 & 0.01771 & 2,3 \\
\hline 25 & 190.79630 & 5241.192 & 0.04227 & 1 & 53 & 473.57292 & 2111.607 & 0.04240 & 1 \\
\hline 26 & 199.43287 & 5014.219 & 0.75646 & 1 & 54 & 507.17014 & 1971.725 & 0.02086 & 2,3 \\
\hline 27 & 201.85648 & 4954.015 & 0.14059 & 2 & 55 & 519.68866 & 1924.229 & 0.02130 & 1 \\
\hline 28 & 209.17477 & 4780.691 & 0.03207 & 1 & 56 & 542.17014 & 1844.439 & 0.01896 & 1 \\
\hline
\end{tabular}

Notes. The amplitudes correspond to the amplitudes of the highest frequency peaks found in multiplets (based on the frequency/amplitude fits to all data).
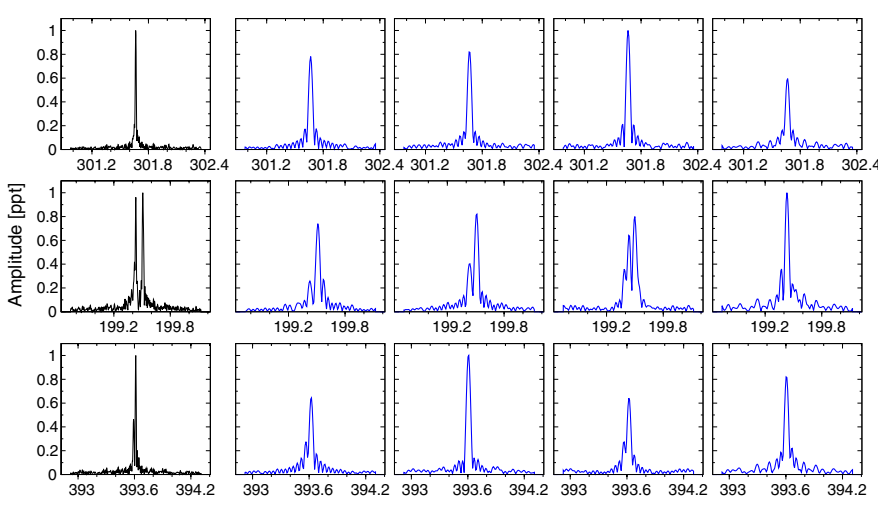

Frequency $[\mu \mathrm{Hz}]$

Fig. 2. Three highest amplitude $l=1 \mathrm{~g}$-modes. Left panels show all data FT, panels on the right present season 1, 2,3, 4 data FTs. Note the change in amplitudes and multiplet component visibility. Amplitudes are normalized by the maximum amplitude of the all data FT in the left panels and by maximum multiplet component amplitudes occurring during seasonal data in the right panels.

excitation, one could use multiplet amplitudes to determine the orientation of the rotation axis in space as done by Charpinet et al. (2011) for KIC 5807616 star. However, hot subdwarfs are not stochastically driven, and the equipartition of energy between multiplet component amplitudes fails (e.g. Pablo et al. 2012, see their Figs. 2 and 5).

In Fig. 2 we show the three highest amplitude (according to the frequency and amplitude fits to the whole time series data)

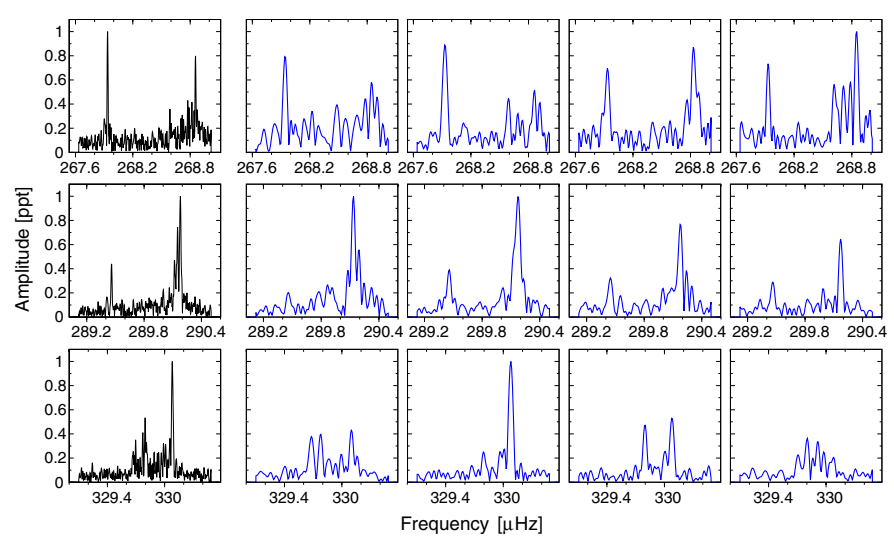

Fig. 3. Best examples of $l=2 g$-modes. None of the multiplets show all multiplet components excited at the same time. Amplitudes are normalized the same way as in Fig. 2. Widths of all panels in Figs. 2 and 3 are the same $(1.4 \mu \mathrm{Hz})$.

$l=1 g$-modes. Two of them have single peaks visible in their whole data FT, but seasonal FTs in the middle panels of Fig. 2 reveal an unstable triplet structure. More convincing are three examples of the best $l=2 g$-modes (Fig. 3). Their multiplets do not have full $\mathrm{m}$ componentes excited, but at least two components are visible in every one of them. One can note that the excited azimuthal m-orders of these modes are different in each presented mode, which contradicts our expectations coming from linear theory, that $\mathrm{m}$ componentes are equally excited. In both Figs. 2 and 3 FTs we can see that most of the multiplet 

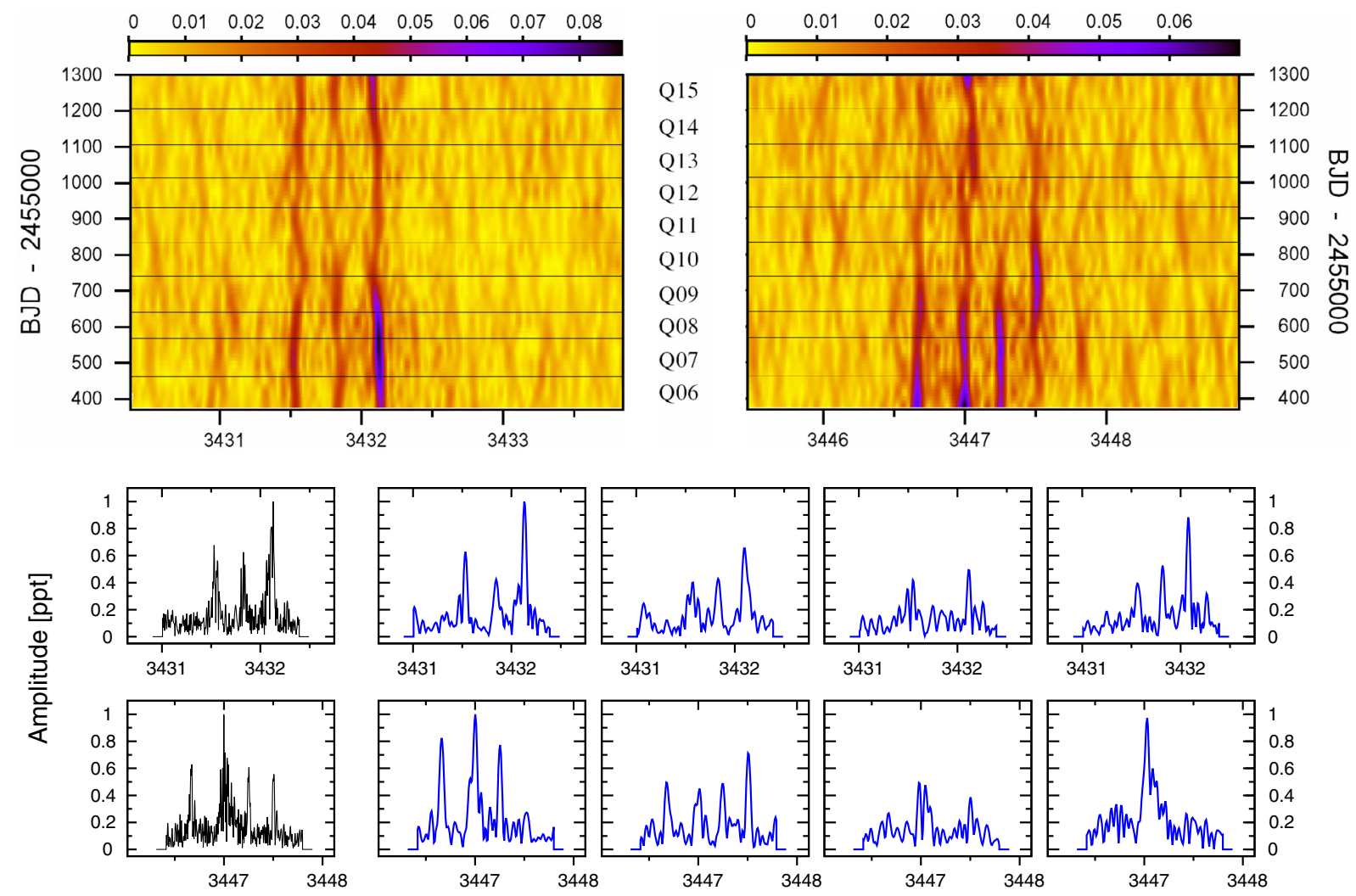

Frequency $[\mu \mathrm{Hz}]$

Fig. 4. Top panel: 200 days running FT of the whole data near 3431.8383 and $3447.2453 \mu \mathrm{Hz}$ showing amplitude and phase changes of the two $p$-modes. Both running FT plots have the same width of $3.5 \mu \mathrm{Hz}$. The two bottom panels show the ordinary FT of the same frequency regions: all data FT (the first panel on the left) and FT of season 1,2,3,4 data (next four panels on the right). Amplitudes were normalized by the largest amplitude of all seasons.

$m$ component amplitudes change greatly: some of them disappear completely, while others grow in amplitude. Taking this into account and having more data to prove it, we show that the average amplitude ratios of any multiplet $m$ componentes will be different for different seasons and will lead to non-consistent conclusions. Therefore, the inclination of the rotation axis of the star cannot be determined this way.

\section{Multiplets and rotation}

The rotation of a star lifts degeneracy in azimuthal order, creating multiplets. The frequencies of the multiplet components will be separated in the frequency domain following the asymptotic relations: $v_{g_{n, l, m}}=v_{g_{n, l, 0}}+m \cdot\left(1-C_{n, l}\right) \cdot \frac{1}{P_{\text {rot }}}$, for $g$-modes where $P_{\text {rot }}$ is the rotation period and $C_{n, l} \sim \frac{1}{l(l+1)}^{\text {rot }}$ is the Ledoux coefficient (Ledoux 1951). For $p$-modes, the Ledoux coefficient is very small, and multiplet components split into $v_{p_{n, l, m}}=$ $v_{p_{n, l, 0}}+m \cdot \frac{1}{P_{\text {rot }}}$ frequencies. Since KIC 5807616 pulsates in both $p$ and $g$-modes, we can use these relations for mode identification and to determine the stellar rotation rate. Although, in case of KIC 5807616, the task is difficult since in the $g$-mode frequency region we do not see well-defined multiplets. Instead, we observe rapid seasonal changes in multiplet appearances (Figs. 2 and 3). The FTs of the seasonal data as well as running FTs show constant amplitude and phase change of multiplet components. Because amplitude variation results in generating sidelobes in the frequency domain, while power flow between components manifests as a frequency and phase change, the multiplets split into forests of many frequencies in the final FTs. In this regard $g$-modes of KIC 5807616 cannot be used in a simple way as a probe of the stellar rotation. However, in the $p$-mode frequency region, there are two multiplets at 3431.8383 and $3447.2453 \mu \mathrm{Hz}$ (Fig. 4), which were stable for about $\sim 500$ days. We identified them as $l=1$ and $l=2$ modes. Table 2 contains their component frequencies and amplitudes fitted to all and to seasonal data, as well as our multiplet component identifications.

The $p$-modes show clear multiplets, even in the whole data FT, but to derive their splitting, we used frequencies fitted to the seasonal data (Table 2) and a histogram of the frequency separation between consecutive components of all multiplets vs. separation occurrence numbers. In Fig. 5 we present an averaged sum of ten $p$-mode splitting histograms, calculated using the bin width of $0.01 \mu \mathrm{Hz}$ and bin centre shifts of $0.001 \mu \mathrm{Hz}$. The splitting read from the histogram maximum is $0.258 \pm 0.006 \mu \mathrm{Hz}$, which is lower then the $0.295 \mu \mathrm{Hz}$ splitting derived earlier by Charpinet et al. (2011).

We also attempted to derive $g$-mode splittings from similar histograms as described above, but made separately for identified $l=1$ and $l=2$ modes (Fig. 6). In this case, however, we used frequencies fitted to the whole data instead of the seasonal data. Because $g$-mode multiplets do not have clear structures, we predicted a theoretical $g$-mode splitting (assuming solid body rotation) from $0.258 \mu \mathrm{Hz}$ splitting of the two $p$-modes and estimated the widths of $g$-mode multiplets. The predicted values of $l=1$ and $l=2 g$-mode splittings are 0.129 and $0.215 \mu \mathrm{Hz}$ respectively. This translates into $\sim 0.258 \mu \mathrm{Hz}$ (for $l=1$ ) and $\sim 0.86 \mu \mathrm{Hz}$ (for $l=2$ ) widths of $g$-mode multiplets. In Fig. 6 
Table 2. Two clear $p$-mode component frequencies.

\begin{tabular}{|c|c|c|c|c|c|}
\hline \multicolumn{2}{|c|}{ Mode } & \multirow{2}{*}{$\begin{array}{c}\text { Frequency } \\
{[\mu \mathrm{Hz}]}\end{array}$} & \multirow{2}{*}{$\begin{array}{r}\text { Period } \\
{[\mathrm{s}]}\end{array}$} & \multirow{2}{*}{$\begin{array}{l}\text { Amplitude } \\
\text { [ppt] }\end{array}$} & \multirow[t]{2}{*}{$S / N$} \\
\hline$l$ & $m$ & & & & \\
\hline \multirow{14}{*}{1} & $(-1$ & 3431.5296 & 291.42 & $0.0204(24)$ & 4.7 \\
\hline & & s1 $3431.5315(26)$ & & $0.0412(45)$ & 7.2 \\
\hline & & s2 $3431.5730(37)$ & & $0.0274(44)$ & 4.8 \\
\hline & & s3 $3431.5512(37)$ & & $0.0292(48)$ & 5.1 \\
\hline & & s4 $3431.5610(41)$ & & $0.0251(46)$ & 4.4 \\
\hline & 0 & 3431.8274 & 291.39 & $0.0200(24)$ & 4.6 \\
\hline & & s1 3431.8479 (39) & & $0.0270(45)$ & 4.7 \\
\hline & & s2 $3431.8317(33)$ & & $0.0310(44)$ & 5.4 \\
\hline & & s4 $3431.8180(29)$ & & $0.0359(46)$ & 6.2 \\
\hline & +1 & 3432.12996 (44) & 291.36 & $0.0310(24)$ & 7.1 \\
\hline & & s1 $3432.1311(15)$ & & $0.0692(45)$ & 12.0 \\
\hline & & s2 $3432.0970(21)$ & & $0.0482(44)$ & 8.4 \\
\hline & & s3 $3432.1145(32)$ & & $0.0343(48)$ & 6.0 \\
\hline & & s4 $3432.0769(17)$ & & $0.0599(47)$ & 10.4 \\
\hline \multirow{14}{*}{2} & $(-2$ & 3446.6725 & 290.13 & 0.0217 (24) & 5.0 \\
\hline & & s1 $3446.6624(20)$ & & $0.0527(45)$ & 9.2 \\
\hline & & s2 $3446.6797(33)$ & & 0.0306 (44) & 5.3 \\
\hline & $\mid-1$ & $3446.99920(38)$ & 290.11 & $0.0357(24)$ & 8.2 \\
\hline & & s1 $3447.0000(17)$ & & $0.0634(45)$ & 11.0 \\
\hline & & s2 $3447.0102(37)$ & & $0.0270(44)$ & 4.7 \\
\hline & & s3 3446.9835 (35) & & 0.0308 (48) & 5.4 \\
\hline & & s4 3447.0291 (17) & & $0.0618(46)$ & 10.7 \\
\hline & 0 & 3447.2453 vis & 290.09 & 0.018 & 4.1 \\
\hline & & s1 $3447.2546(21)$ & & $0.0504(45)$ & 8.8 \\
\hline & & s2 $3447.2475(30)$ & & 0.0336 (44) & 5.8 \\
\hline & +1 & 3447.5072 & 290.06 & $0.0200(24)$ & 4.6 \\
\hline & & s2 $3447.5076(21)$ & & $0.0472(44)$ & 8.2 \\
\hline & & s3 3447.5014 (46) & & $0.0237(48)$ & 4.1 \\
\hline
\end{tabular}

Notes. Dashes mean no detection, vis - indicates a frequency not fitted but added to the list after a visual inspection of the FT. Frequency and amplitude fitting errors are given in () brackets.

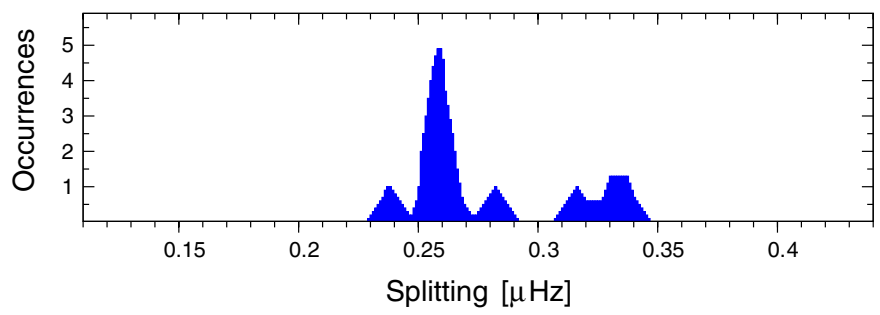

Fig. 5. Averaged histogram of $p$-mode frequency separation based on two multiplets at 3431.8383 and $3447.2453 \mu \mathrm{Hz}$ during seasons $1,2,3$, and 4.

we show histograms of multiplet component frequency separation in broader ranges (top two panels) than predicted multiplet widths to accommodate for possible deviations from predicted values. The bottom panel of Fig. 6 shows the same, but overlapped, histograms ( $l=1$ black and $l=2$ green $)$ presented within the range of $l=1$ mode splitting. As one can see, the histograms have weak multiple and scattered maxima. The frequency separation maxima of $l=1$ and $l=2$ modes are interspersed, which might be caused by wrong mode identification. Although from an eye inspection, we know that some of $l=2$ multiplets have additional and closer components than expected for $l=2$ modes, therefore, we did not change mode identification based on the histograms.

The splitting for $l=1 g$-modes read from the histogram is $0.106 \mu \mathrm{Hz}$. For $l=2$ modes we have to use an eliminating
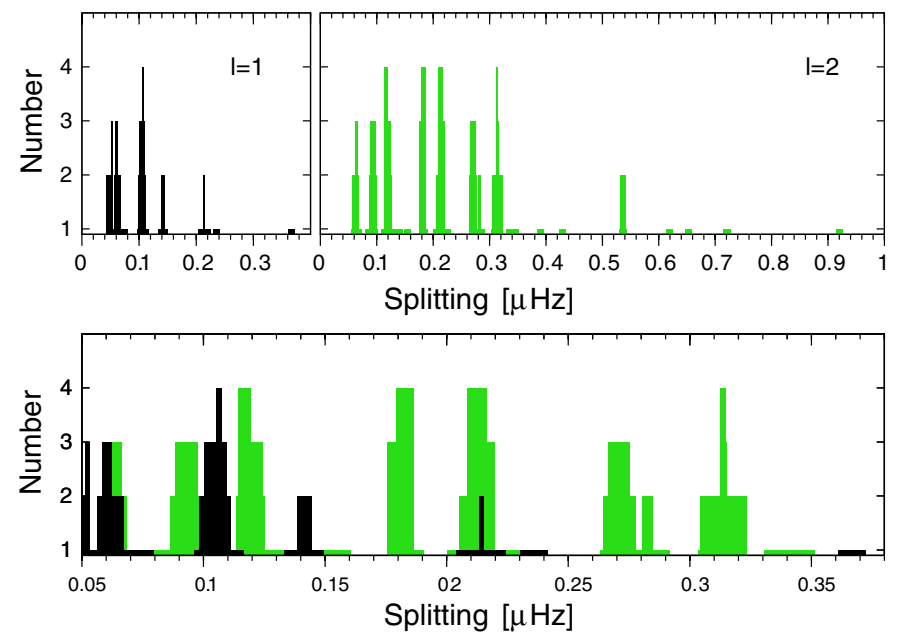

Fig. 6. Histogram of $l=1$ (top left panel) and $l=2$ (top right panel) $g$-mode frequency separations. The bottom panel shows the enlarged region of the $l=1$ histogram overlapped with the histogram of $l=2$ modes frequency separations.

procedure. For example, the peak at $0.212 \mu \mathrm{Hz}$ (bottom panel of Fig. 6) is very close to double that of the $0.106 \mu \mathrm{Hz}$ splitting, while two separation peaks around $0.1 \mu \mathrm{Hz}$ are likely fake splittings or made by accidently included $l=1$ mode into the $l=2$ modes sample. The separation peak, which might be considered as a real $l=2$ modes splitting is the one at $0.183 \mu \mathrm{Hz}$. Assuming Ledoux constant (see above) $\mathrm{C}_{l=1}=0.5$ and $\mathrm{C}_{l=2}=0.1667$, the theoretical ratio of $l=1$ to $l=2$ modes frequency splittings should be 0.6 . The ratio of the frequency splittings read from our histogram $(0.106 / 0.183=0.58)$ is nearly the same as the theoretical one. Both $l=1$ and $l=2$ mode splittings are lower than 0.129 and $0.215 \mu \mathrm{Hz}$ splittings derived from $p$-mode multiplets.

Since multiplets are created by the rotation of the star, we can calculate the rotation rate from multiplet frequency splittings. Using the above equations for multiplet splitting and the most reliable $0.258 \mu \mathrm{Hz} p$-mode splitting, we can determine the rotation period as $44.9 \pm 1.1$ days. This places KIC 5807616 within slowly rotating sdB stars. The longest, $\sim 90$ day rotational period, was recorded for KIC 10670103 (Krzesinski et al. 2014).

Similarly, from $g$-mode splittings we would have $54.6 \pm 4.1$ and $52.7 \pm 3.2$ days rotation rates for $l=1$ and $l=2$ modes. However, taking multiple maxima of the histograms into account (Fig. 6) and rather weak statistics (we have about 4 occurrences at each histogram maximum out of 56 identified modes e.g.. $35 l=1$ and $21 l=2$ modes) we do not consider $g$-mode splitting determination to be conclusive.

\section{Planets or not planets}

In the low frequency range Charpinet et al. (2011) found two frequencies at 33.839 and $48.182 \mu \mathrm{Hz}$ (planetary signature frequencies). After careful analysis of Q5-Q8 data and using 61.02 and $105.69 \mu \mathrm{Hz}$ pulsation cut-off limits of $l=1$ and $l=2 g$-modes respectively, Charpinet et al. (2011) interpreted 33.839 and $48.182 \mu \mathrm{Hz}$ peaks in the low frequency region as a result of the light reflected by planets orbiting the KIC 5807616 sdBV star. The observed complex structure around $33.839 \mu \mathrm{Hz}$ was explained as possible evidence of a third body in the system dynamically perturbing the planet at the $33.839 \mu \mathrm{Hz}$ orbital frequency. 


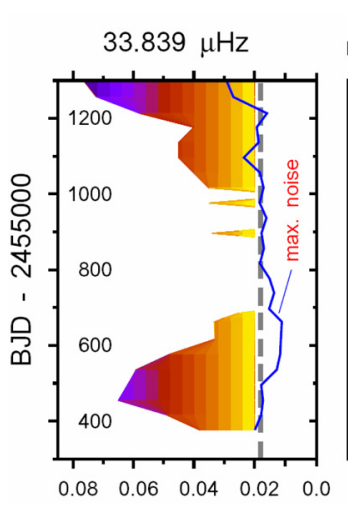

Amplitude [ppt]

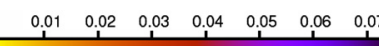

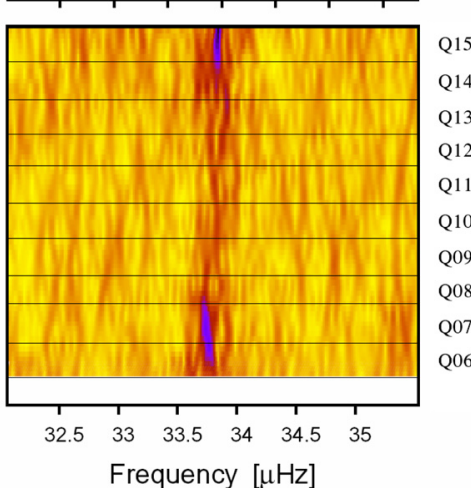

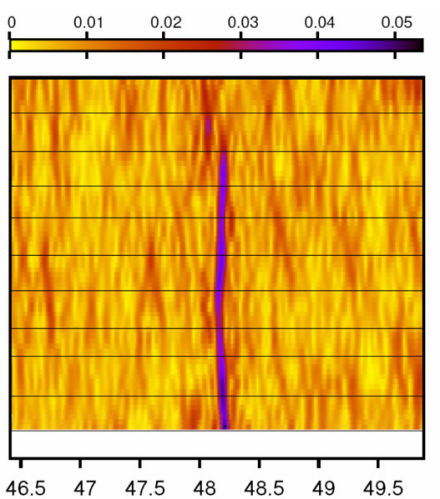

Frequency $[\mu \mathrm{Hz}]$

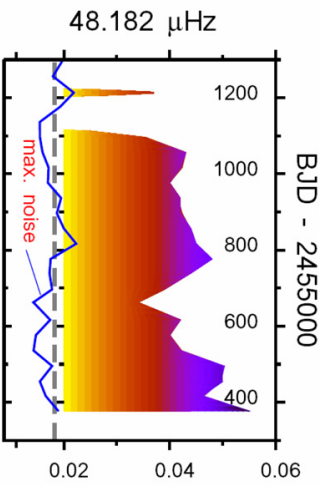

Amplitude [ppt]

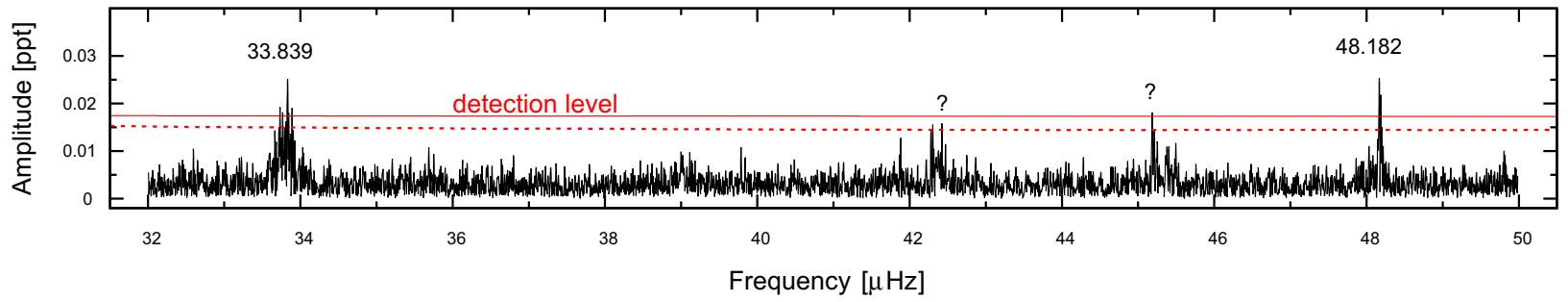

Fig. 7. 200-day running FTs of the whole data (upper panels) and FT (bottom panel) near two frequencies at $33.839 \mu \mathrm{Hz}$ and $48.182 \mu \mathrm{Hz}$. Both running FT plots have the same width of $3.5 \mu \mathrm{Hz}$. Side upper panels show amplitude changes during Q 5-Q 17. Thick dashed lines in the amplitude panels mark the $4 \sigma$ detection threshold applied in this work $(0.01743 \mathrm{ppt})$, while continuous lines denote the maximum amplitude of background noise near both frequencies. In the bottom panel the dotted line (below the solid one) shows the local detection threshold $(0.01549 \mathrm{ppt})$.

Here, using the new Kepler data set we were able to analyse these frequencies and amplitude change over a period of time that is three times longer than the analysis done before and found some problems with previous interpretations of the signals.

Table 3 contains frequencies within $33-49 \mu \mathrm{Hz}$ fitted to all and seasonal data, while in Fig. 7 we present high resolution (200 days) running FT windows of $3.5 \mu \mathrm{Hz}$ width around 33.839 and $48.182 \mu \mathrm{Hz}$ and the amplitude variations of these two frequencies during Q 5-Q17. The signal peak amplitudes for the plot were calculated along the running FT in narrow bands of $\pm 0.4 \mu \mathrm{Hz}$ around the planetary signature frequencies. If more than one peak was found, the amplitude of the highest peak was taken. Similarly, the maximum noise was calculated as the maximum noise out of the noise levels taken from two $0.5 \mu \mathrm{Hz}$ width strips in the proximity of both planetary signature frequencies.

The running FT, as well as the FT of the whole data (bottom part of Fig. 7), shows complex structures around 33.839 and $48.182 \mu \mathrm{Hz}$ frequencies. As already mentioned, similar structures in the frequency domain can be generated by the amplitude and phase changes in the signal, and this is what happens to our planetary signature frequencies (Fig. 7 and Table 3). Both, 33.839 and $48.182 \mu \mathrm{Hz}$ frequencies are not constant in amplitudes, as assumed in the previous work. The peak at $33.839 \mu \mathrm{Hz}$ goes beyond the detection level in the middle of the Kepler run, i.e. after Q9 (note that Charpinet et al. (2011) analysis only includes Kepler data up to Q 8), while the $48.182 \mu \mathrm{Hz}$ peak goes beyond the detection level at the end of the run. Since one would expect a constant amplitude of the FT signal during the whole observation period, if it is due to the light reflected by the orbiting planets, then change in this amplitude cannot be explained by the reflection effect. In terms of the signal-tonoise ratio, both frequencies have small amplitudes that vary between non-detection $(S / N<4)$ up to 6 or 8 , but the evidence that both amplitudes and frequencies are unstable is convincing. We also note two low amplitude frequencies at 42.3 and
Table 3. Planetary signature frequency region between $33-49 \mu \mathrm{Hz}$.

\begin{tabular}{|c|c|c|c|c|}
\hline $\begin{array}{c}\text { Group } \\
\text { no }\end{array}$ & $\begin{array}{c}\text { Frequency } \\
{[\mu \mathrm{Hz}]}\end{array}$ & $\begin{array}{c}\text { Period } \\
{[\mathrm{s}]}\end{array}$ & $\begin{array}{c}\text { Amplitude } \\
\text { [ppt] }\end{array}$ & $S / N$ \\
\hline \multirow{7}{*}{1} & 33.7429 (7) & 29635.9 & $0.0189(24)$ & 4.3 \\
\hline & 33.9019 (7) & 29496.9 & $0.0190(24)$ & 4.4 \\
\hline & s1 33.6036 (32) & & $0.0334(46)$ & 4.9 \\
\hline & s1 $33.7488(24)$ & & $0.0433(46)$ & 6.4 \\
\hline & s2 $33.8396(36)$ & & $0.0274(44)$ & 4.1 \\
\hline & s3 $33.7877(38)$ & & $0.0283(48)$ & 4.2 \\
\hline & s4 $33.8483(15)$ & & 0.0677 (46) & 10.0 \\
\hline \multirow{4}{*}{2} & $* 42.3174(8)$ & 23630.9 & $0.0162(24)$ & 4.3 \\
\hline & $* 42.4374(9)$ & 23564.1 & $0.0160(24)$ & 4.3 \\
\hline & $\mathrm{s} 1 \quad 42.3095(38)$ & & 0.0275 (45) & 4.1 \\
\hline & s2 $42.3958(36)$ & & $0.0281(44)$ & 4.2 \\
\hline \multirow{2}{*}{3} & $45.2008(8)$ & 22123.5 & $0.0180(24)$ & 4.1 \\
\hline & s3 $45.1884(35)$ & & $0.0307(48)$ & 4.5 \\
\hline \multirow{4}{*}{4} & $48.1823(6)$ & 20754.5 & $0.0252(24)$ & 5.8 \\
\hline & s1 $48.2070(20)$ & & $0.0537(45)$ & 8.0 \\
\hline & s2 $48.1659(23)$ & & 0.0433 (44) & 6.4 \\
\hline & s3 $48.1890(27)$ & & $0.0400(48)$ & 5.9 \\
\hline
\end{tabular}

Notes. Frequencies belonging to the same group (multiplet) of frequencies are indicated by curly brackets. Large fonts denote frequencies fitted to all data, while small fonts denote frequencies fitted to seasonal data (if detected above the local seasonal detection threshold of $0.027 \mathrm{ppt}$ ) and labelled with s1, s2, s3, s4 in the second column. Asterisks mark frequency amplitudes below all data $0.01743 \mathrm{ppt}$ detection threshold, but above the local one (see Fig. 7). Fitting errors are given in () brackets.

$45.2 \mu \mathrm{Hz}$ (see question marks in Fig. 7, asterisks in Table 3), which likely have a similar structure and behaviour to the above planetary signature frequencies, but their $\mathrm{S} / \mathrm{N}$ is too low to make any certain conclusions. However, if these were signals caused 
by two additional planets around KIC 5807616, that would raise questions about dynamical stability of the whole close-in planet system.

The Kepler data is known to have an artificial frequency presence (artefacts, Baran 2013) in FTs of the light curves (the list of known artefacts can be found on MAST web pages). If a frequency is found below the pulsation cut-off frequency and is not on the artefact list, we often call it an unknown artefact or we try to find other explanations for its existence. However, the cut-off frequency formula given by Hansen et al. (1985) was derived for white dwarfs and involved some approximations.

For example, the pulsation cut-off frequencies derived for an Eddington grey atmosphere (Hansen et al. 1985, Eqs. (8a) and (8b)) depend on the mean molecular weight $\mu, \log g$, and on the effective temperature of the star. If one assumes for the KIC 5807616 sdBV star the same mean molecular weight as for white dwarfs $(\mu=1)$, the $l=1$ and $l=2$ mode cut-off frequencies will be at 31.28 and $54.18 \mu \mathrm{Hz}$, respectively. This is about half the 61.02 and $105.69 \mu \mathrm{Hz}$ limits derived by Charpinet et al. (2011), and allows for both planetary signature frequencies to be identified as pulsating $g$-modes. Decreasing the mean molecular weight down to 0.6 will raise the cut-off frequencies up to $\sim 40$ and $\sim 70 \mu \mathrm{Hz}$. Therefore, final result strongly depends on the assumptions made to derive the cut-off frequencies for stars other than white dwarfs.

We might also need to reconsider our approach to the strict limits of the cut-off frequency, because damping does not necessarily mean that pulsations have to stop beyond it. It simply means that the amplitude of pulsation will be lower and might be difficult to detect. This could be the case for KIC 5807616 (and a couple of other stars), where a few frequencies were detected below the cut-off frequency. They were not found on the list of known Kepler spacecraft artefacts, and we argue they are not planetary signatures either. Therefore, we postulate these are simply pulsation frequencies, which are damped and of low amplitudes, but observed below the cut-off frequency.

\section{Conclusions}

We performed the analysis of the whole Q 5-Q 17 Kepler data of the KIC 5807616 sdBV star by means of the FTs and high resolution running FTs of its light curve. The FTs do not show clear multiplets in the $g$-mode frequency region. There are only a few multiplets that have more than one component that is excited and stable for longer periods of time. Therefore, in spite of a large number $(\sim 191)$ of frequencies fitted to the star light curve, we were able to identify only 56 pulsation $g$-modes $(35 l=1$ and 21 $l=2$ modes) in a narrow 2000-12000 s period range (Table 1).
The lack of clear multiplets also makes our identification less reliable.

In the $p$-mode frequency region, we found two clear multiplet structures that were identified as acoustic $l=1$ and $l=2$ modes. These were used to derive a $44.9 \pm 1.1$ days rotation rate of the star envelope/surface, which is five days longer than previous estimates by Charpinet et al. (2011). From the analysis of $g$-mode splitting, the rotation rate seems to be about ten days longer, suggesting different surface and interior rotation rates. However, our $g$-mode multiplet-splitting determination is not reliable so this value has to be used carefully.

Finally, we used frequency splittings and amplitude variations of the two 33.839 and $48.182 \mu \mathrm{Hz}$ frequencies to challenge the interpretation that these signals are from planets around KIC 5807616. We also suggest that the pulsation cut-off frequencies should not be treated as strict limits below which one cannot observe pulsating modes. Pulsations might be strongly damped but still visible in the FTs of the light curve. Since the Kepler data were carefully reduced and there are no known artefact frequencies within this region, we concluded that both planetary signature frequencies (and perhaps a couple of others) observed beyond the cut-off frequency limits are most likely of pulsation origin. The example of KIC 5807616 also has implications for other claims of extreme planetary systems around sdB stars based on similar assumptions of the strict cut-off frequency limits for the pulsating modes (Silvotti et al. 2014, KIC 10001893 $\mathrm{sdBV}$ ), which might need to be reanalysed in a similar way to how it has been done in this paper.

Acknowledgements. This project was supported by Polish National Science Centre grant 2011/03/D/ST9/01914.

\section{References}

Baran, A. S. 2013, Acta Astron., 63, 203

Baran, A. S., Pigulski, A., Kozieł, D., et al. 2005, MNRAS, 360, 737

Charpinet, S., Fontaine, G., Brassard, P., et al. 2011, Nature, 480, 496

Charpinet, S., Van Grootel, V., Brassard, P., et al. 2013, EPJ Web of Conferences, 43, 4005

Dziembowski, W. 1977, Acta Astron., 27, 1

Hansen, C. J., Winget, D. E., \& Kawaler, S. D. 1985, ApJ, 297, 544

Krzesinski, J., Blokesz, A., Baran, A. S., \& Bachulski, S. 2014, Acta Astron., 64, 151

Ledoux, P. 1951, ApJ, 114, 373

Østensen, R. H., Silvotti, R., Charpinet, S., et al. 2010, MNRAS, 409, 1470

Pablo, H., Kawaler, S. D., Reed, M. D., et al. 2012, MNRAS, 422, 1343

Randall, S. K., Fontaine, G., Brassard, P., \& Bergeron, P. 2005, ApJS, 161, 456

Reed, M. D., Kawaler, S. D., Østensen, R. H., et al. 2010, MNRAS, 409, 1496

Schuh, S., Huber, J., Green, E. M., et al. 2005, ASP Conf. Ser., 334, 530

Silvotti, R., Charpinet, S., Green, E., et al. 2014, A\&A, 570, A130

Van Grootel, V., Green, E. M., Randall, S. K., et al. 2010, ApJ, 718, L97 